\title{
ELECTRONIC SPECTRA OF CARBON CHAINS OF RELEVANCE TO ASTROPHYSICS
}

\author{
JOHN P. MAIER \\ Institute for Physical Chemistry, University of Basel, \\ Klingelbergstrasse 80, CH-4056 Basel, Switzerland
}

\begin{abstract}
The electronic absorption spectra of a variety of carbon chains have been identified in the laboratory. The measurements were carried out in $5 \mathrm{~K}$ neon matrices on mass-selected species. On the basis of the trends evident in the homologous series, it can be predicted in which region absorption bands of the carbon chains are to be expected for the not yet measured longer and isoelectronic species. The spectroscopic characteristics, photostability and structural considerations point out which and what size carbon chains could be the diffuse interstellar bands absorbers.
\end{abstract}

\section{Introduction}

The long-standing discussion as to the origin of the diffuse interstellar bands (DIBs, Herbig 1995) has motivated us to understand the spectroscopy of carbon chains and their isolectronic species involving nitrogen and hydrogen. To this end we have developed a technique which allows the measurement of the electronic absorption spectra of mass-selected species in $5 \mathrm{~K}$ neon matrices (Forney et al. 1995a). This has now been applied to the study of carbon chains, $\mathrm{C}_{2 n} n=2-5, \mathrm{C}_{2 n} \mathrm{H} n=3-8$ (Freivogel et al. 1995, 1996), $\mathrm{C}_{2 n+1} n=2-7$ (Forney et al. 1996), $\mathrm{HC}_{2 n+1} \mathrm{H} n=2-7$ (Fulara et al. 1995), of their ions $\mathrm{C}_{2 n}^{-} n=2-10$ (Freivogel et al. 1995), $\mathrm{HC}_{n} \mathrm{H}^{+}$ $n=4-16$ (Freivogel et al. 1994; Fulara et al. 1995), as well as to some of the related nitrogen-containing ions, $\mathrm{HC}_{n} \mathrm{~N}^{+} n=5-13$ and $\mathrm{NC}_{n} \mathrm{~N}^{+}$ $n=4-12$ (Forney et al. 1995b).

With this information in hand, one can draw some conclusions on the relevance of such species to the DIBs by combining the spectroscopic results with the astrophysical observations and physical restrictions. These aspects are outlined here. It should also be emphasized that the development of the 
technique combining mass-selection with matrix isolation has the aim of eventually measuring such spectra in the gas-phase. This is now a realistic proposition because the electronic transitions have been located.

\section{Structural constraints of carriers}

If one considers the type of molecules which could be responsible for the DIBs, then these criteria should be considered. 1) They should have the right spectroscopic characteristics - strongly allowed transitions in the range of observed DIBs (400-900 nm). 2) They should have sufficiently high abundances - be either large enough (say $>10-15$ atoms) to be photostable in the (inter)stellar radiation field or be synthesised efficiently (Thaddeus 1994; Bettens \& Herbst 1995). 3) The number of structurally and chemically possible species should be restricted in view of the fact that there appears to be a limited number of stronger DIBs in the $400-900 \mathrm{~nm}$ region (Jenniskens \& Désert 1994).

The species which have attracted a lot of attention in this connection have been the PAH molecules and their ions (Puget \& Léger 1989). A drawback to these, from a spectroscopist's view point, is that criterion 3 ) is not satisfied, since an enormous number of possible derivatives of $\mathrm{PAH}$ and their ions would contribute to the absorption features. In contrast, this is not the case for the carbon chains. In the ensuing discussion carbon chains refer not only to the $\mathrm{C}_{n}, \mathrm{C}_{n}^{-}$and $\mathrm{C}_{n}^{+}$species, but also to their mono and dihydro derivatives and their ions, as well as to the isoelectronic and related compounds containing nitrogen and oxygen. Although not all the possible species have yet been studied, the available information can be used to draw inferences on the spectroscopic properties of the isoelectronic species and their relevance to the DIB problem.

\section{Laboratory spectra}

The carbon chains were obtained by producing ions in an appropriate source - cesium sputter source for the bare carbon anions, or hot-cathode discharge for the cations and anions containing one or two hydrogens. After mass-selection the species were co-deposited with an excess of neon to form a thin matrix at $5 \mathrm{~K}$. If the mass-selected neutral species were studied, the ion beam was neutralized during or following deposition. The absorption spectra were measured in the $200-1000 \mathrm{~nm}$ region by a waveguide approach and further in the infrared by a reflection method.

The details of the procedures used can be found in the references given to the published studies. There also the reasons for the assignment of the spectra to specific electronic transitions can be found. These are based 


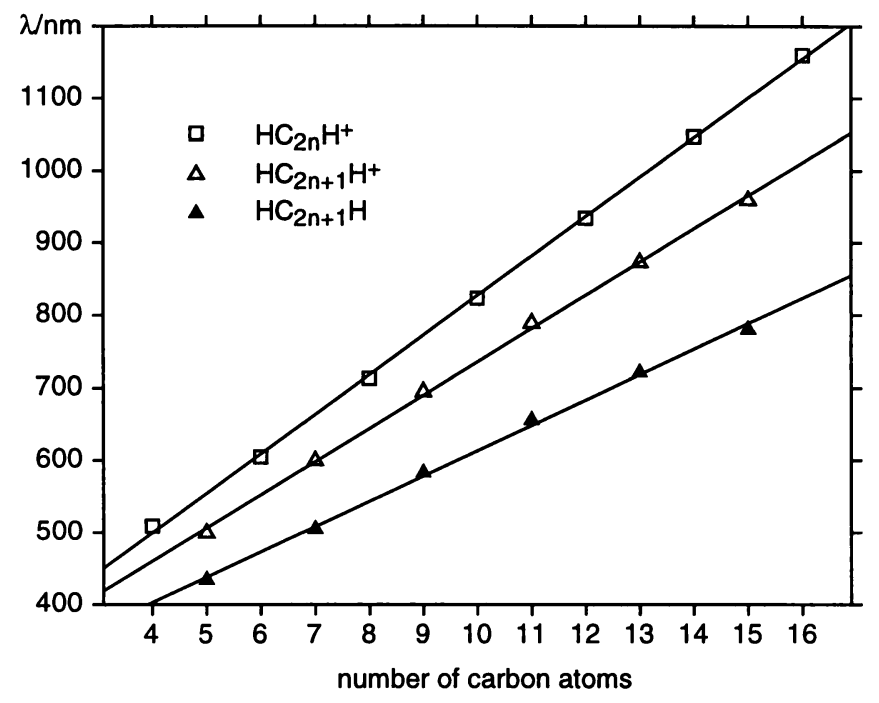

Figure 1. Wavelength dependence of the origin band of the $\pi \leftarrow \pi$ electronic transitions of $\mathrm{HC}_{2 n} \mathrm{H}^{+}, \mathrm{HC}_{2 n+1} \mathrm{H}^{+}$and $\mathrm{HC}_{2 n+1} \mathrm{H}$ on the number of carbon atoms in the chain.

on the experimental observations, theoretical calculations on some of the smaller species and spectroscopic trends along homologous series.

\section{Characteristics of the electronic spectra}

The electronic spectra of the polyacetylene cations, $\mathrm{HC}_{2 n} \mathrm{H}^{+}$, are taken as typical example (Freivogel et al. 1994). These species with an odd-number of electrons have a ${ }^{2} \Pi$ ground state and show a strong $A^{2} \Pi \leftarrow X^{2} \Pi$ transition. The origin of this band system shifts in a regular manner to lower energies as the number of carbon atoms increases, about $110 \mathrm{~nm}$ for each additional two carbon atoms. In Figure 1 the position of the origin band of this transition is shown for the series $n=4$ to 8 . The inverse dependence of the energy of the transition on the length of the chain is evident, in accord with the simple concept of a particle in a one-dimensional box. This treatment also indicates that the oscillator strength of the transition is proportional to the number of carbon atoms, explaining why the longer chains can be detected even though their concentration is successively smaller with increasing size. This appealing attribute has not escaped notice in relation to astrophysical observations (Watson 1994).

These electronic transitions of the species $\mathrm{HC}_{2 n} \mathrm{H}^{+} n=2,3,4$ have in fact been detected in the gas-phase by the observation of their emission spectra (Allan et al. 1976). The origin bands are shifted by 79, 135 and 143 $\mathrm{cm}^{-1}$ to the blue of the values in the neon matrix, respectively. 
It can be seen from Figure 1 that only four polyacetylene cations $\mathrm{HC}_{2 n} \mathrm{H}^{+}$ $n=2-5$ would absorb in the $400-900 \mathrm{~nm}$ DIB range. The question is whether these species are large enough to be resistant to photodissociation in the diffuse cloud environments. The longer species will have their electronic absorptions in the infrared region. In contrast, the neutral counterparts, $\mathrm{HC}_{2 n} \mathrm{H}$, are closed shell species, with singlet ground states and absorb in the 200-400 $\mathrm{nm}$ region for $n=2-5$ (Kloster-Jensen et al. 1974). In order for the neutral polyyenes to absorb in the visible, they would have to have more than twenty carbon atoms. However, it has been experimentally demonstrated that carbon species comprising more than 20 atoms prefer ring structures (Gotts et al. 1995) which may place an upper limit to the length of the chains to be considered.

In Figure 1 the data are included for the $\mathrm{HC}_{2 n+1} \mathrm{H}$ chains and their cations, whose absorption spectra have been measured. In the DIB region, species with 17 atoms absorb.

\section{Homologous series of carbon chains}

The series of carbon chains which are likely to be easily formed are: $\mathrm{C}_{n}$; $\mathrm{C}_{n}^{+} ; \mathrm{C}_{n}^{-} ; \mathrm{C}_{n} \mathrm{H} ; \mathrm{C}_{n} \mathrm{H}^{+} ; \mathrm{C}_{n} \mathrm{H}^{-} ; \mathrm{HC}_{n} \mathrm{H}, \mathrm{HC}_{n} \mathrm{H}^{+}$(or their $\mathrm{H}_{2} \mathrm{C}_{n}$ isomers). One can also construct a number of isoelectronic (and related) species with $\mathrm{N}$ (O); e.g. $\mathrm{CH}$ can in each case be replaced by $\mathrm{N}$. Which of these should be considered as possible DIB carriers? Though not all these homologous series have been studied, we have learned enough about the electronic transitions from the investigated groups to exclude some on spectroscopic grounds, and others on stability criteria (both photophysical and chemical). The series studied hitherto are the $\pi-\pi$ transitions of $\mathrm{C}_{2 n+1}\left({ }^{1} \Sigma_{u}^{+} \leftarrow X^{1} \Sigma_{g}^{+}\right), \mathrm{C}_{2 n}$ and $\mathrm{HC}_{2 n+1} \mathrm{H}\left({ }^{3} \Sigma_{u}^{-} \leftarrow X^{3} \Sigma_{g}^{-}\right), \mathrm{C}_{2 n} \mathrm{H}, \mathrm{C}_{2 n}^{-}, \mathrm{HC}_{n} \mathrm{H}^{+}, \mathrm{NC}_{n} \mathrm{~N}^{+}$and $\mathrm{HC}_{n} \mathrm{~N}^{+}$ $\left({ }^{2} \Pi \leftarrow X^{2} \Pi\right)$.

Consider the bare carbon chains. The even ones, $\mathrm{C}_{6}$ to $\mathrm{C}_{10}$ will absorb in the 400-900 $\mathrm{nm}$ region (Freivogel et al. 1995). On the other hand, the odd numbered species have the corresponding $\pi-\pi$ transition to higher energy; in Figure 2 are shown the absorption spectra of the $\mathrm{C}_{9}-\mathrm{C}_{15}$ species (Forney et al. 1996). Again one sees the characteristic monotonic trend; the shift to the red is around $43 \mathrm{~nm}$ for each additional two carbon atoms. Now it is usually assumed that only sufficiently large species will withstand the stellar uv radiation field; sizes with 10-15 atoms have been considered as minimum (Leach 1995). Extrapolating this leads to the conclusion that it will be the species $C_{15}-C_{37}$ in size which will be absorbing in the $400-900$ nm DIB range. 


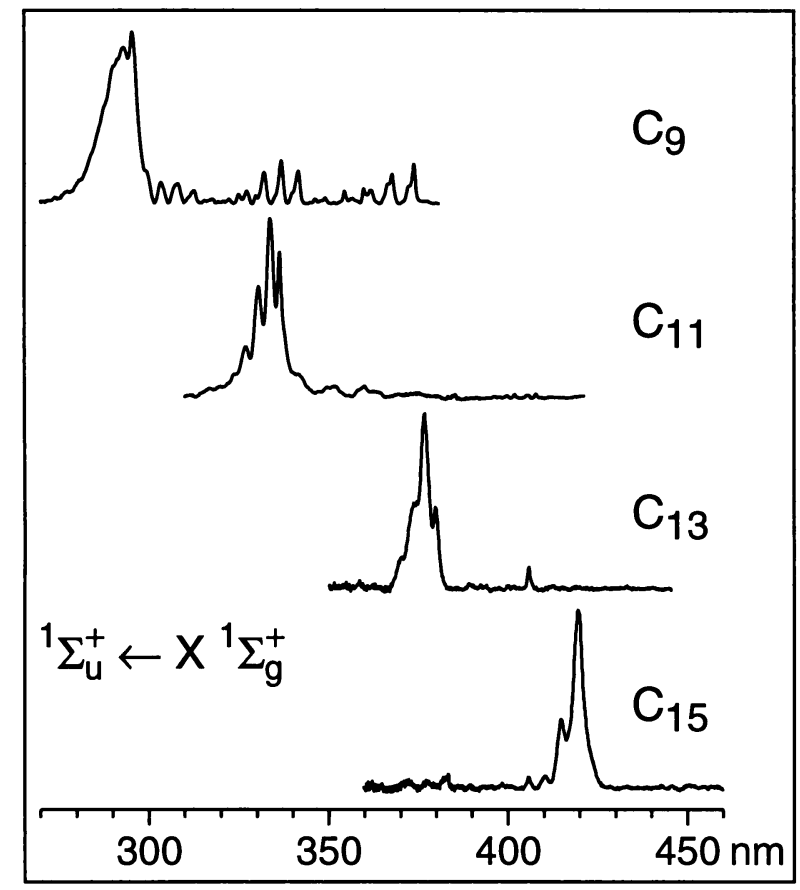

Figure 2. Absorption spectra of the electronic transitions $\left({ }^{1} \Sigma_{u}^{+} \leftarrow X^{1} \Sigma_{g}^{+}\right)$of mass-selected carbon chains in $5 \mathrm{~K}$ neon matrices. The weaker bands lying above $300 \mathrm{~nm}$ in the $\mathrm{C}_{9}$ spectrum are due to a forbidden transition.

\section{Astronomical measurements}

Even though gas-phase values of the origin bands of the carbon chains studied are not known for a direct comparison with DIBs, there is already sufficient information available to make specific searches. The gas-phase values are expected to be blue shifted relative to the matrix values in the 50-100 $\mathrm{cm}^{-1}$ range for chains with 10-15 atoms. Possible candidates are $\mathrm{C}_{10}(736 \mathrm{~nm}), \mathrm{C}_{10} \mathrm{H}(722 \mathrm{~nm}), \mathrm{C}_{12} \mathrm{H}(801 \mathrm{~nm}), \mathrm{C}_{14} \mathrm{H}(866 \mathrm{~nm}), \mathrm{HC}_{9} \mathrm{H}(583$ $\mathrm{nm}), \mathrm{HC}_{11} \mathrm{H}(656 \mathrm{~nm}), \mathrm{HC}_{13} \mathrm{H}(722 \mathrm{~nm}), \mathrm{HC}_{15} \mathrm{H}(781 \mathrm{~nm}), \mathrm{HC}_{8} \mathrm{H}^{+}(713$ $\mathrm{nm}), \mathrm{HC}_{9} \mathrm{H}^{+}(695 \mathrm{~nm}), \mathrm{HC}_{10} \mathrm{H}^{+}(823 \mathrm{~nm}), \mathrm{HC}_{11} \mathrm{H}^{+}(789 \mathrm{~nm})$ and $\mathrm{HC}_{13} \mathrm{H}^{+}$ $(873 \mathrm{~nm})$, the wavelengths being those of the origin bands in a neon matrix.

Thus one selects DIBs which lie within $100 \mathrm{~cm}^{-1}$ of the matrix values. Then DIBs which lie to shorter wavelength by an energy amount which corresponds to the separation of the peaks in the absorption spectrum in the neon matrix should be searched for (Freivogel et al. 1994). This difference is the frequency of the vibrations in the excited electronic state. Because the vibrational frequencies are known not to differ by more than $5-10 \mathrm{~cm}^{-1}$ from the gas-phase values, the uncertainty in the location of a 
DIB is reduced to this amount.

If such a DIB is found, it then has to satisfy two further criteria 1) the half-widths of the DIB pair should be similar and 2) the ratio of their equivalent widths should be comparable to the intensity ratio of the two bands in the matrix absorption spectrum. This procedure has been proposed (Maier 1994) to test the suggestion that two DIBs in the near infrared are due to $\mathrm{C}_{60}^{+}$(Foing \& Ehrenfreund 1994) .

\section{Other isoelectronic series}

Species which are isoelectronic are expected to have transitions in a similar spectral region. Thus one can infer where these should be for species not yet studied. As an example consider the $\pi-\pi$ transitions of the four isolectronic series $\mathrm{C}_{2 n}, \mathrm{HC}_{2 n} \mathrm{H}^{+}, \mathrm{C}_{2 n} \mathrm{H}$ and $\mathrm{HC}_{2 n-1} \mathrm{~N}^{+}$studied. In Figure 3 the measured spectra for these chains with $n=3$ are shown. The transitions lie near to each other energetically, show a similar band intensity distribution and comparable vibrational frequencies.

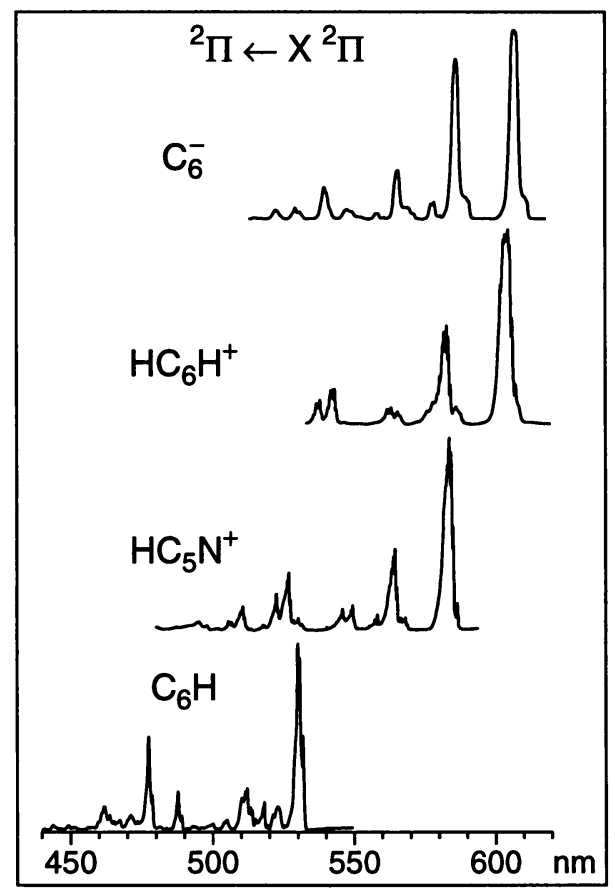

Figure 3. Electronic absorption spectra $\left({ }^{2} \Pi \leftarrow X^{2} \Pi\right.$ transition) of isoelectronic species observed in $5 \mathrm{~K}$ neon matrices using mass-selection.

Thus the hitherto unmeasured spectra of $\mathrm{HC}_{2 n+1}^{+}$will follow the pattern of the isoelectronic odd-numbered bare carbon chains $\mathrm{C}_{2 n+1}$ (Figure 2) and 
again the species with $n=7-18$ will show strong transitions in the 400$900 \mathrm{~nm}$ spectral window. The same applies to the also isoelectronic $\mathrm{C}_{2 n} \mathrm{~N}^{+}$ species. On the other hand the neutral $\mathrm{C}_{2 n} \mathrm{~N}$, and $\mathrm{HC}_{2 n+1}$ chains can be compared to observations made on the $\pi-\pi$ transitions of the isoelectronic $\mathrm{HC}_{2 n+1} \mathrm{H}^{+}$, the results for which can be seen in the plot of Figure 1, and can be extrapolated accordingly.

\section{Possible DIB carriers}

In view of the spectroscopic knowledge gained, and adopting the concept that the minimum size for photostability in the diffuse clouds are species with 10-15 atoms, then the homologous series which would have strong transitions in the $400-900 \mathrm{~nm}$ region are the odd carbon chains, $\mathrm{C}_{2 n+1}$ and their isoelectronic analogues, $\mathrm{HC}_{2 n+1}^{+}$and $\mathrm{C}_{2 n} \mathrm{~N}^{+}$. In addition, it appears that there is a natural upper limit for the size of the linear chains because a variety of experiments as well as theoretical calculations have shown that for around $n>20$ ring-like structures are preferred. These would then have different, yet unknown, spectroscopic properties.

Thus the chains to be considered have as upper bound in the midtwenties for the number of carbon atoms. This restricts the number of possible species, in line with the curent observations that there appear to be hundred or so stronger DIBs. The structurally related carbon chains including nitrogen and oxygen atoms would also have similar spectral and size restrictions $\left(\right.$ e.g $\left.\mathrm{C}_{n} \mathrm{O}\right)$.

It may be relevant, that the inferred rotational constants of order $3.6 \times$ $10^{-3} \mathrm{~cm}^{-1}$ or smaller, inferred from the structure resolved in recent high resolution measurements of DIBs (Ehrenfreund \& Foing 1996), corresponds in case of the carbon chains to the species of $\mathrm{C}_{15}$, and larger, in size.

\section{Acknowledgements}

The studies in Basel have been financed by the Swiss National Science Foundation (project no. 20-41768.94).

\section{References}

Allan, M., Kloster-Jensen., E., Maier, J.P., 1976, Chem. Phys. 7, 11

Bettens. R.P.A., Herbst, E., 1995, Int.J.Mass Spectrom.Ion Processes 150, 321

Ehrenfreund, P., Foing, B.H., 1996, A\&A 305, L25

Foing, B.H., Ehrenfreund, P. 1994, Nature 369, 296

Forney, D., Fulara, J., Freivogel, P., Jakobi, M., Lessen, D., Maier, J.P., 1995a, J. Chem. Phys. 103, 48

Forney, D., Freivogel, P., Fulara, J., Maier, J.P., 1995b, J. Chem. Phys. 102, 1515

Forney, D., Freivogel, P., Grutter, M., Maier, J.P., 1996, J. Chem. Phys. 104, 4954

Freivogel, P., Fulara, J., Lessen, D., Forney, D., Maier, J.P., 1994, Chem. Phys. 189, 335 
Freivogel, P., Fulara, J., Maier, J.P., 1994, ApJ 405, L151

Freivogel, P., Fulara, J., Jakobi, M., Forney, D., Maier, J.P., 1995, J. Chem. Phys. 103, 54

Freivogel, P., Grutter, M., Forney, D., Maier, J.P., 1996, Chem. Phys. Lett. 249, 191

Fulara, J., Freivogel, P., Forney, D., Maier, J.P., 1995, J. Chem. Phys. 103, 8805

Gotts, N.G., von Helden, G., Bowers, M.T., 1995, Int.J.Mass Spectrom. Ion Processes 150,217

Herbig, G.H., 1995, Ann. Rev. Astron. Astrophys. 33, 19

Jenniskens, P., Désert, F-X., 1994, A\&A Suppl. 106, 39

Kloster-Jensen, E., Haink, H-J., Christen, H., 1974, Helv. Chim. Acta 57, 1731

Leach., S., 1995, in The Diffuse Interstellar Bands, eds. A.G.G.M. Tielens and T.P. Snow, (Dordrecht; Kluwer), p.281

Maier, J.P., 1994, Nature 370, 423

Puget, J.L., Leger, A., 1989, A\&A 27, 161

Thaddeus, P., 1994, in Molecules and Grains in Space, ed. I. Nenner (New York: AIP Press), p.711

Watson, J.K.G., 1994, ApJ 437, 678

\section{Discussion}

Ubachs: Did you try to find coincidences between frequency separations between DIBs and the lines in spectra of the molecules recorded, e.g. $\mathrm{C}_{12} \mathrm{H}$, by a computer search?

Maier: Yes, we looked for coincidences, though not by computer search. The difficulty encountered was the following: We chose DIBs lying, say, within $\pm 100 \mathrm{~cm}^{-1}$ of the matrix bands and then tried to find DIBs at higher energy, corresponding to vibrationally excited levels as observed in the matrix. However, as these usually have intensities a factor of 5 smaller than the origin bands, they tend to be buried in the noise of the astronomical spectra. One would have to make a specific search in the predicted regions so that the $\mathrm{S} / \mathrm{N}$ would be sufficient to detect DIBs of the expected equivalent width.

Scappini: A. Douglas in 1977 pointed out that a carbon chain has to have a number of atoms larger than $6-8$ to resist photodissociation.

Maier: A. Douglas indeed pointed out that carbon chains of a certain length will be stable to photodissociation due to internal energy conversion. Specifically he suggested ones in the $\mathrm{C}_{n} n=5-15$ range.

Scappini: What is the lifetime of an ion in a matrix?

Maier: An ion can be kept in the matrix for long periods; in its ground state it is stable there.

Takano: Did you measure cyclic forms of carbon-chain molecules? For example, $\mathrm{C}_{4}$ is predicted to have linear and cyclic forms at nearly the same energy according to ab initio calculations. Are there unidentified bands in your spectra?

Maier: We have not yet been able to identify transitions of cyclic forms. Firstly, we favour the linear species because our source of anions effectively anneals them. Under such conditions the ratio of linear to cyclic anions is increased. Secondly, often we are near detection limits and the linear species are easier to observe. With improved sensitivity and other ion sources, we hope to be able to observe the cyclic species. 